\title{
A Cooperative Spectrum Sensing with Multi-Agent Reinforcement Learning Approach in Cognitive Radio Networks
}

\author{
Ang Gao, Chengyuan Du, Soon Xin Ng, Wei Liang
}

\begin{abstract}
Cognitive radio networks (CRNs) can greatly improve the temporal and spatial spectrum utilization by identifying and exploring spectrum holes of the licensed primary users (PUs). However, since the occupation of primary channels changes dynamically, a swift and accurate spectrum sensing is crucial especially in the multi-channel multi-secondary users (SUs) environment, where the number of channels is much larger than that of SUs. To improve the sensing accuracy, a cooperative sensing algorithm is proposed in this paper, where multiple SUs can share their spectrum detection results for a more effective spectrum holes search. This paper further employs multi-agent deep deterministic policy gradient (MADDPG) algorithm with the feature of centralized training and decentralized execution to reduce the synchronization and communication overhead caused by the sensing cooperation of SUs. The numerical simulation demonstrates that with the combination of cooperative sensing and multi-agent reinforcement learning, the proposed algorithm can greatly enhance the sensing accuracy in comparison to other non-cooperative learning or centralized learning approaches.
\end{abstract}

Index Terms-Cognitive Radio Networks, Cooperative Spectrum Sensing, Deep Reinforcement Learning, Multi-Agent Deep Deterministic Policy Gradient

\section{INTRODUCTION}

C OGNITIVE radio networks (CRNs) are envisaged to solve the spectrum inefficiency by exploring the existing wireless spectrum holes opportunistically. The primary users (PUs) and secondary users (SUs) coexist in CRNs, where PUs mainly present in certain geographic areas and specific frequency bands with short transmission duration, while SUs opportunistically transmit in the vacant portion of the spectrum already assigned to the licensed PUs. Since CRNs have no pre-established infrastructure to manage and coordinate the transmitting task in central, SUs should effectively identify and explore the spectrum holes of the primary network for quickly accessing. However, since the sensing accuracy is susceptible to the channels fading and shadowing effect, SUs have to repeatedly sense the licensed channels until they find an available channel or fail, which results in low spectrum efficiency and time-wasting.

Cooperative spectrum sensing (CSS) has been proposed to overcome the performance degradation (e.g. caused by multipath fading and shadowing) without increasing the transceivers complexity [1]. Given the spectrum concerned is wide and

The work was supported by China and Shaanxi Postdoctoral Science Foundation 2017M623243, 2018BSHYDZZ26, Shaanxi and Guangxi Keypoint Research and Invention Program 2019ZDLGY13-02-02, AB19110036, and Taicang Keypoint Science and Technology Plan TC2018SF03,TC2019SF03. (Corresponding author:Wei Liang, e-mail:liangwei@nwpu.edu.cn)

Ang Gao, Chengyuan Du and Wei Liang are with the School of Electronics and Information, Northwestern Polytechnical University, 710072, China.

Soon Xin $\mathrm{Ng}$ is with the School of Electronics and Computer Science, University of Southampton, Southampton SO17 1BJ, U.K. non-consecutive, it will be more efficient if each SU only senses a part of the spectrum once a time. On this basis, SUs can find the "vacant position" cooperatively and occupy the unused spectrum bands to transmit information temporarily by only partial observation of the frequency band [2], [3], [4].

Although CSS can largely enhance the accessing efficiency of SUs, there are still some challenges. First, the topology of CRNs changes dynamically because the nodes are mobile and will join or leave the network randomly [1]. Second, the change of channels state will affect the cooperation strategy of SUs. Third, PUs' action is hardly to predict. Although hidden Markov model (HMM) has been used to predict the future channels state, it highly depends on the spectrum usage distribution [5]. To solve the issues above, the paper proposes a multi-agent deep deterministic policy gradient (MADDPG) based approach to investigate CSS in multi-SU CRNs. The main contributions are as follows:

- By partners selection and channels state fusion algorithm, each SU can be global aware of the recent primary channels, and the one-time success probability achieves about $90 \%$ after well-trained. Since SUs can access the primary channels directly rather than sense-and-access opportunistically, the temporal and spatial spectrum utilization can be enhanced.

- MADDPG is adopted to overcome the drawback of traditional deep reinforcement learning (DRL) algorithms such as Q-learning and deep Q-learning network (DQN), which perform poorly in multi-SU CRNs due to their non-stationary in complex environment [6]. With the feature of centralized training and distributed execution of MADDPG, each SU can individually select a vacant channel for transmission by partial observation, which greatly reduces the synchronization and communication overhead caused by cooperative spectrum sensing.

\section{SYSTEM MODEL}

Suppose that there are $M$ PUs, $N$ SUs and $N_{f b}$ orthogonal channels in a CRN, where SUs can opportunistically access the available channels without interfering PUs. The transmitting power of PUs is fixed to $p$, and the channels noise is Additive White Gaussian Noise (AWGN) with a power of $\delta_{0}^{2}$.

In general, PUs have the first priority to occupy the available channels. Channels occupation can be modeled by a twostate dynamic Markov chain [7], i.e. active and inactive state. SUs can only use the channels in inactive state. Let $\boldsymbol{O}(t)=\left[o_{1}(t), \cdots, o_{N_{f b}}(t)\right]$ be the channels state vector at time slot $t$, where $o_{f}(t)=1$ means that the channel is vacant and 


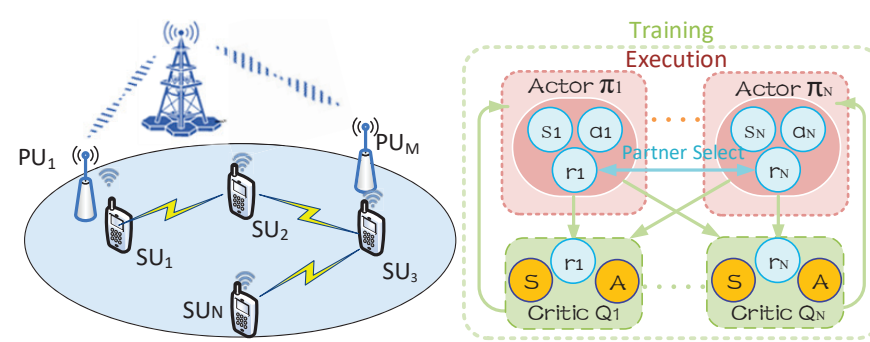

Fig. 1: System model

$o_{f}(t)=0$ means that the channel is occupied by PUs. The transition probability of $\boldsymbol{P}_{f}$ is given by:

$$
\boldsymbol{P}_{f}=\left[\begin{array}{ll}
p_{0,0}, & p_{0,1} \\
p_{1,0}, & p_{1,1}
\end{array}\right],
$$

where $p_{1,0}$ is the transition probability from a vacant (inactive) state to an occupied (active) state.

Suppose that channels follow the quasi-static block fading, i.e. channels state remains unchanged in a transmission frame. Before the secondary transmitting, a SU needs to sense all the primary channels one by one until finds an idle channel. Or the transmission is considered to be failed if none idle channel can be found after a certain sensing period. This procedure may lead to a high sensing and accessing overhead, especially when the number of channels or frequency bands is significantly larger than the number of SUs [3].

Since the sensing accuracy is affected by the shadowing and multi-path fading effect, a single SU is incapable of detecting multiple channels simultaneously. However, multiuser cooperative sensing could provide a way to combat the problem, by which SUs can communicate and share the channels state with each other through a specific control channel. Let $\mathcal{G}_{n}$ be the partners set. SU in the set can update its channel state to select a channel with the maximum vacant probability. Actually, SUs don't know the state of PUs and the occupancy pattern of the primary channels. They have to learn from their historical sensing results and from their partners.

\section{A. Reinforcement Learning Framework}

The actor-critic (AC) framework is adopted by SUs to interact with the environment and to learn the best sensing action from their historical mistakes. The aim is to find the channel with the maximum probability to be vacant. Then SUs can take the vacant channel for immediate secondary communication, without scanning the whole band or retrying for many times.

As shown in Fig. 1, each SU works as an independent agent to interact with the environment and to make an action according to its own policy.

- Each SU maintains a set of channels state $\mathbf{s}_{n}(t)=$ $\left[b_{n, 1}, \cdots, b_{n, N_{f b}}\right]$, where $b_{n, f} \in[0,1]$ denotes the belief of the $n^{\text {th }}$ SU on channel $f$ whether it is vacant or occupied, and relates to its own action and channels belief from other SUs.

- When the $n^{\text {th }}$ SU initiates a call, it will make a sensing action $a_{n}[t] \in \mathcal{N}$ to seek a vacant channel, i.e. to determine which spectrum band to sense at time slot $t$.
- Reward $r_{n}(t)$ is returned from the environment to critic the action $a_{n}(t)$. A positive value will be returned if $a_{n}(t)=$ $f$ and channel $f$ is detected to be vacant. Otherwise it returns a negative value. The sensing result can be used to update the channels belief and transit to the next state $\mathbf{s}_{n}(t+1)$.

$$
r_{n}\left(\mathbf{s}_{n}, a_{n}\right)=\left\{\begin{array}{lr}
1, & a_{n}(t) \text { channel is vacant } \\
-1, & a_{n}(t) \text { channel is occupied }
\end{array}\right.
$$

The goal of the multi-agent learning algorithm is to maximize the expected sum of future rewards for each agent:

$$
R^{n}(t)=\mathbb{E}\left[\sum_{t^{\prime}=0}^{\infty} \gamma^{t^{\prime}} r_{n}\left(t+t^{\prime}\right)\right]
$$

where $\gamma<1$ is the discount factor.

\section{B. Multi-Agent DDPG based Deep Learning}

MADDPG [8] scheme is adopted for each agent to learn the channels state, i.e. the channel with the maximum probability is supposed to be vacant. MADDPG inherits DDPG [6] method into the multi-agent domain. It not only eliminates the non-stationary feature of $Q$-learning and policy gradient, but also reserves the great advantage of DDPG that can search the action space in a continuous rather than discrete way.

As shown in Fig. 1, each agent has an actor and a critic, where the actor maps the local observation state $\mathbf{s}_{n}$ into a proper action $a_{n}$ according to the policy network $\pi_{n}$, while the critic evaluates the fitness of such policy according to its value network $Q_{n}$. Both the actor and critic have a online and a target network to ensure the learning stability and overcome the overoptimistic [6].

During the training, $Q_{n}$ outputs the policy gradient for $\pi_{n}$ with the ensemble policies of other agents as $\mathcal{A}(t)=\left[a_{n}\right]$ and the global state as $\mathcal{S}(t)=\left[\mathbf{s}_{n}\right]$. During the execution, the welltrained $\pi_{n}$ can make the optimal action $a_{n}$ based on its own state $\mathbf{s}_{n}$ independently without interfering with other agents' state, which leads to less synchronization and communication overhead.

MADDPG further adds noise $\mathbb{N}$ to explore better strategies in the training process. Another essential technique is the experience replay buffer (RB). Each agent is equipped with a RB to store the (state, action, reward, next state) transition $\left[\mathcal{S}(t), \mathcal{A}(t), r_{n}(t), \mathcal{S}(t+1)\right]$, which will be fetched out randomly to update the weight. Together with mini-batch, experience replay can effectively avoid the highly correlated action for successive updating.

\section{Belief Fusion and Decay}

As aforementioned, state $\mathbf{s}_{n}(t)$ is the channels belief that depends on the historical sensing results, not only from its own but also from other SUs. However, such belief will become uncertain along with time because the channels occupation changes dynamically. So the channels belief sensed by each SU should be fused with others and decay with time.

- The belief of a specific channel $f$ is deterministic once it has been sensed, i.e. $\bar{b}_{n, f}=1$ for vacancy and $\bar{b}_{n, f}=0$ for occupation. Such belief will decay to 0.5 , if the channel 
is not sensed in the adjacent time slot [3]. So the decay rate can be expressed by a forgetting factor $\alpha \in[0.5,1)$. If $\bar{b}_{n, f}>0.5$, SUs would tend to believe that channel $f$ is vacant and vice versa. If $\bar{b}_{n, f}=0.5$, the channel state is totally uncertain.

- To ensure SUs would take the global aware ability of recent channels, the belief of channel $f$ should be a weighted sum with its partners':

$$
b_{n, f}=\sum_{k \in\left\{\mathcal{G}_{n} \cup n\right\}} \omega_{n, k} \bar{b}_{k, f},
$$

where $\mathcal{G}_{n}$ is the cooperative partners set of the $n^{\text {th }}$ $\mathrm{SU}, \omega_{n, k}$ is the corresponding reliable-dependent weight, and the subscript $\cup n$ denotes the current SU itself. The detailed algorithm for partners selection is in Sec. II-D.

For each SU, the channels belief at every time slot is updated according to Algorithm 1,

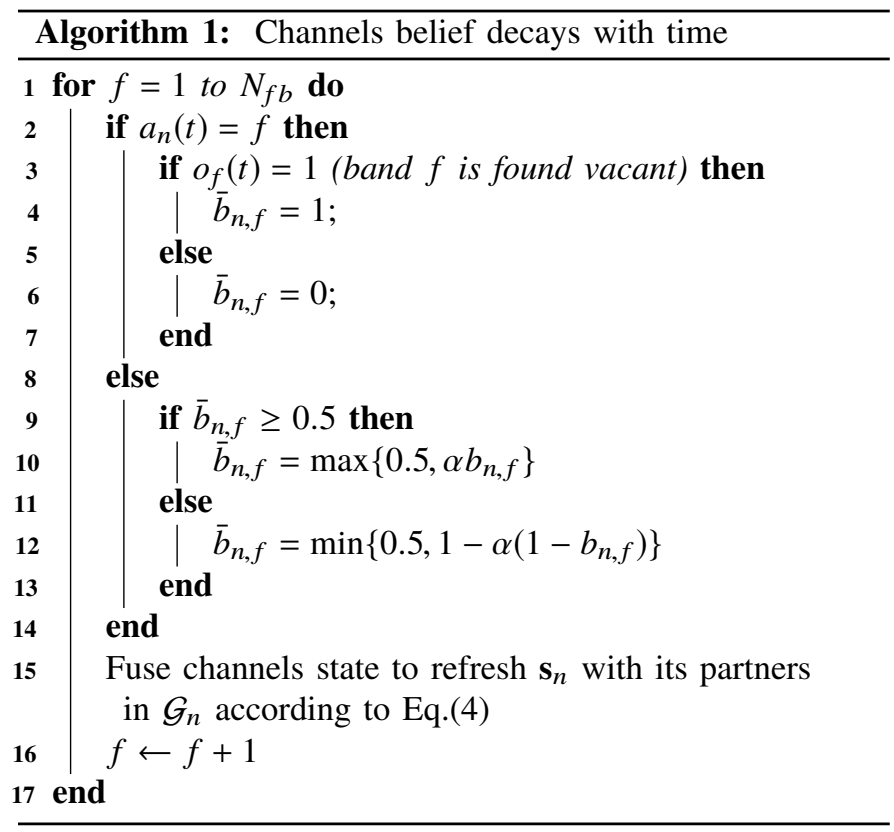

\section{Reliability based Partner Selection}

Due to the different location of SUs and the time varying feature of channels, the sensing ability of SUs changes dynamically and is unknown to others. Thus it is reasonable to set different partners weight $\omega_{n, k}$ according to their reliability from the historical reward.

In every time slot $t$, if SU $k$ is assigned to $\mathrm{SU} n$ as the partner and leads to a larger historical accumulative reward, it is considered to have a better reliability and hence a higher weight $\omega_{n, k}$. Every SU can sort and update its partners set $\mathcal{G}_{n}$ based on the reliability. Thus, the partners selection algorithm with the consideration of detection reliability and exploration of new partners [9] is designed as follows:

- SU $k$ will be selected as SU n's partner in priority, if it can help SU $n$ to attain a better historical reward in the past $t^{s}$ time slots. Let $\rho_{n, k}$ be the corresponding reliability:

$$
\rho_{n, k}(t)=\sum_{t^{\prime}=1}^{t^{s}} \gamma^{t^{\prime}} r_{n}\left(t-t^{\prime}\right) \mathcal{I}\left(k\left(t-t^{\prime}\right) \in \mathcal{G}_{n} \cup n\right),
$$

where $k \in \mathcal{N}$. If SU $n$ selects $\mathrm{SU} k$ as its partner, then $k \in \mathcal{G}_{n}, \mathcal{I}(\cdot)=1$. Otherwise $\mathcal{I}(\cdot)=0$.

- Based on the reliability of SUs in set of $\left\{\mathcal{G}_{n} \cup n\right\}$, the fusion weight in Eq.(4) can be calculated as:

$\omega_{n, k}(t)=\frac{\rho_{n, k}(t)}{\sum_{k^{\prime} \in\left\{\mathcal{G}_{n} \cup n\right\}} \rho_{n, k^{\prime}}(t)}, \omega_{n, k} \in(0,1), k \in\left\{\mathcal{G}_{n} \cup n\right\}$.

- SU who is outside of the set $\mathcal{G}_{n}$ still has the probability to be selected as a new partner. Let $\eta_{n, k}$ be the number of times that SU $k$ becomes the partner of SU $n$ in the past $t^{s}$ time slots:

$$
\eta_{n, k}(t)=\sum_{t^{\prime}=1}^{t^{s}} \mathcal{I}\left(k\left(t-t^{\prime}\right) \in \mathcal{G}_{n}\right) .
$$

SU with a larger historical reliability or less cooperative times will be selected as the new partner, which means the partners selection is proportional to the reliability weight $\omega_{n, k}$ and inverse proportional to $\eta_{n, k}$. Thus, $\mathcal{G}_{n}$ can be updated by:

$$
\mathcal{G}_{n}=\arg _{k \in\{\mathcal{N}\}} \operatorname{sortmax}_{N_{\text {coop }}}\left(\omega_{n, k}+\epsilon \frac{1}{\eta_{n, k}}\right),
$$

where the function $\operatorname{sortmax}_{N_{\text {coop }}}(\cdot)$ returns $N_{\text {coop }}$ number of indices as descent order of $(\cdot), 0 \leq N_{\text {coop }} \leq N-1$ and $\epsilon<1$ is the explore factor.

With the combination of multi-agent learning and partners selection, MADDPG based cooperative spectrum sensing scheme is detailed in Algorithm 2 with the feature of centralized training and decentralized execution. In specific, in the $12^{\text {th }}$ line when "training $=$ True", each agent has to use the global state $\mathcal{S}=\left[\mathbf{s}_{n}\right]$, global action $\mathcal{A}=\left[a_{n}(t)\right]$, and local reward $r_{n}$ to update the corresponding actor and critic network. However, only the local state $\mathbf{s}_{n}$ is needed to generate the action $a_{n}$ during the execution.

\section{NUMERICAL RESULT}

Suppose that there are 3 SUs and 5 PUs randomly distributed in a rectangular area of $200 \mathrm{~m} \times 200 \mathrm{~m}$, and there are $N_{f b}=15$ orthogonal channels within a bandwidth of $1 \mathrm{MHz}$. The transmission power of PU is $40 \mathrm{~mW}$, the average noise power density is $-174 \mathrm{dBm} / \mathrm{Hz}$, and the channels gain follows a log-distance with a path loss-exponent of 3.3. The discount faction and explore factor are $\gamma=0.9$ and $\epsilon=0.8$ respectively. The transition probability of each channel is uniformly distributed as $p_{1,1} \in[0.7,1]$ and $p_{0,0} \in[0,0.3]$, where $p_{1,0}=1-p_{1,1}$ and $p_{0,1}=1-p_{0,0}$. The parameter selection depends on the fact that the utilization of licensed spectrum is low [7]. The actor and critic neural network configuration are listed in Table I and RB is $10 \mathrm{kB}$.

\section{A. Impact of Different Number of Cooperative Partners}

Fig. 2 shows the convergence of MADDPG with the number of partners $N_{\text {coop }}$ increasing from 0 to 2 . When $N_{\text {coop }}=$ 0 , SUs only consider the local channel belief of itself. And when $N_{\text {coop }}=2$, SUs will combine the channels belief of all the rest SUs for action generating. The SUs-PUs collision, SUs collision and overall collision are shown in Fig. 2(a)(c) respectively. While the sensing accuracy defined as the 


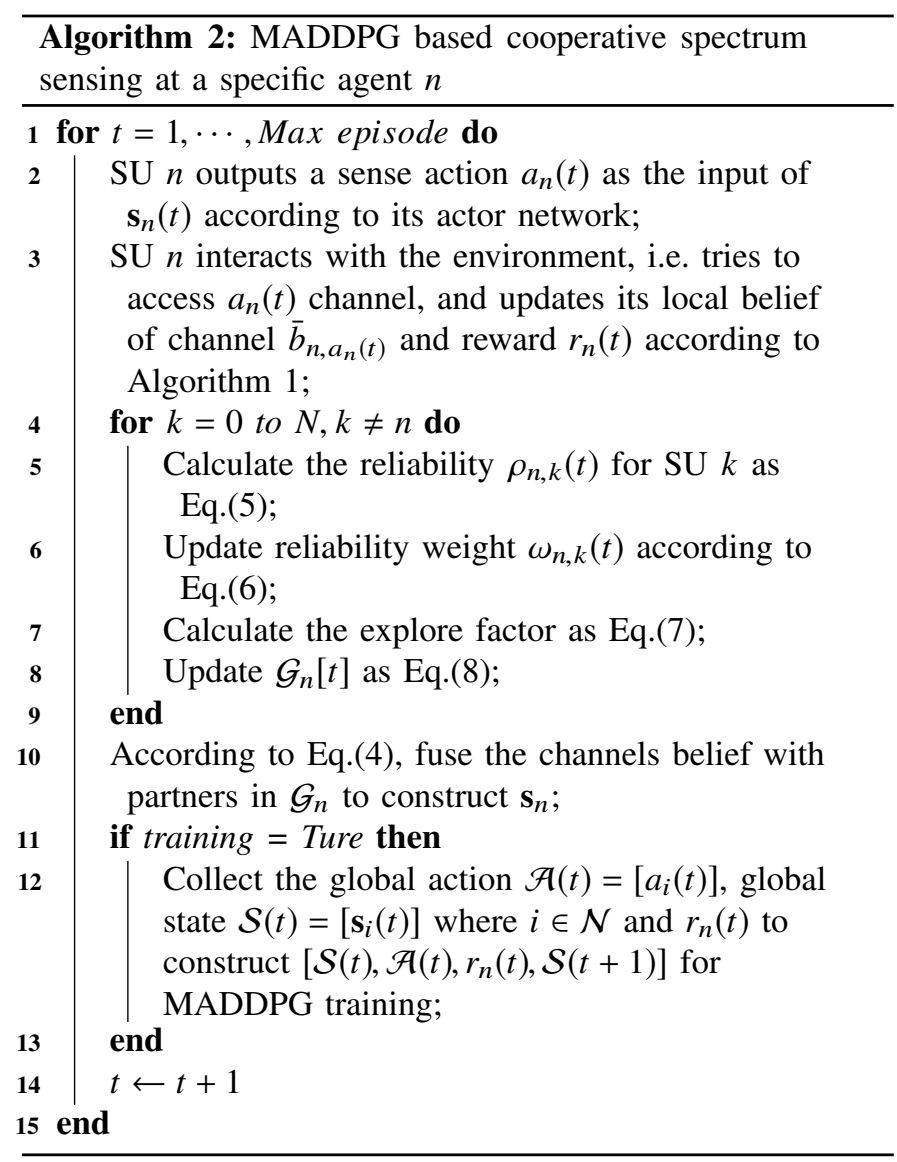

TABLE I: MADDPG neural network configuration.

\begin{tabular}{|c|c|c|}
\hline Name & Neurons Num. and Active Fun. & Type \\
\hline Input layer & $N_{f b}=15$ for $\mathbf{s}_{n}, \operatorname{ReLU}$ & \multirow{3}{*}{ Actor } \\
\hline Hidden Layer & 2 layers with 300 for each layer, ReLU6 & \\
\hline Output layer & 1 for $a_{n}$, Sigmoid & \\
\hline Input layer & $N_{f b} \times N+N \dagger$ for $(\mathcal{S}, \mathcal{A}), \operatorname{ReLU}$ & \multirow{3}{*}{ Critic } \\
\hline Hidden Layer & 2 layers with300 for each layer, ReLU6 & \\
\hline Output layer & $1 \stackrel{\Im}{\mp}, \mathrm{NA}$ & \\
\hline
\end{tabular}

one-time success probability, i.e. sense a vacant channel and access successfully is presented in Fig. 2(d) where success probability $=(1$-overall collision probability $)$.

No matter collision probability or success probability, the performance enhances along with the increasing number of training episode and cooperative partners. Take Fig. 2(d) as an example. The success probability starts from lower than $50 \%$ with 0 to 2 partners. Along with the operation of MADDPG, it converges about $90 \%$ in all three scenario which proves the effectiveness of the proposed algorithm. Meanwhile, the success probability with 2 partners is further enhanced at least $5 \%$ and $3 \%$ respectively compared with those of using no partner and 1 partner.

To evaluate the performance of cooperative sensing with 2 partners, we further design a multi-time accessing scenario, i.e. each SU senses and tries to access a channel more than one time. Once a SU occurs the collision with PUs or SUs, or senses an occupied channel, it would re-select another channel (a) SUs-PUs Collision

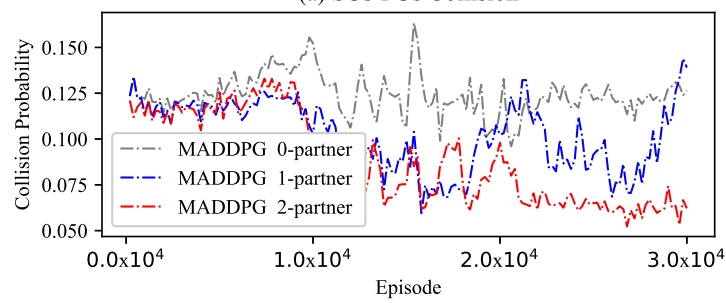

(b) SUs Collision

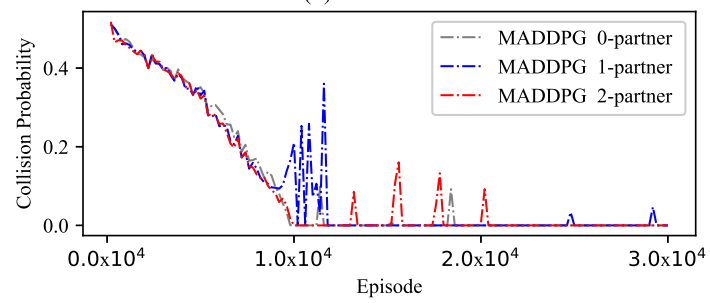

(c) Overall Collision

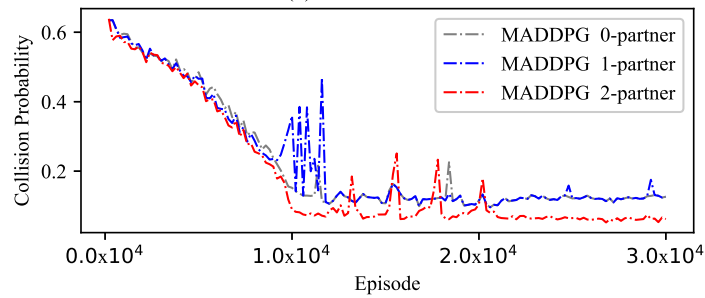

(d) One-Time Success Accessing

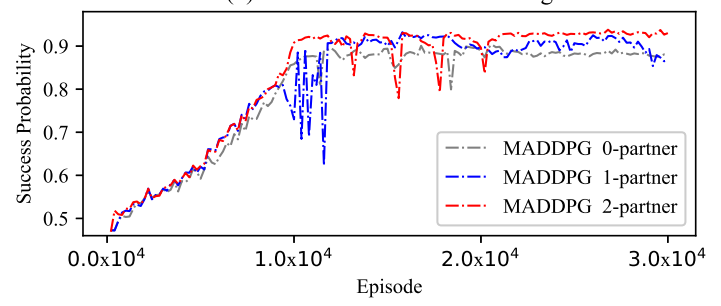

Fig. 2: Convergence of MADDPG with different $N_{\text {coop }}$

to scan intermediately. The sensing will be finished when the SU accesses successfully or fails after 4 times. The detailed result is in Fig. 3, where the one-time success probability blooms from $50 \%$ to nearly $88 \%$.

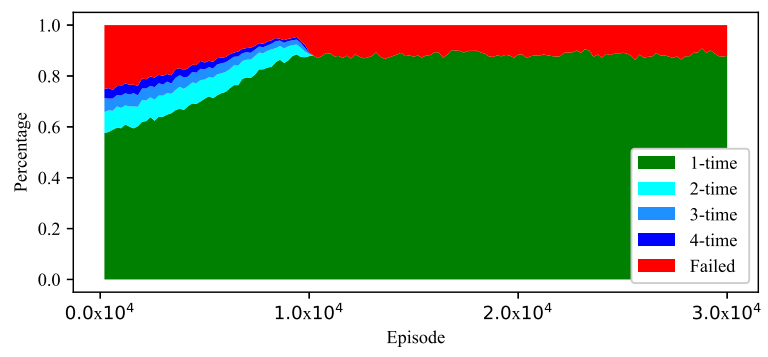

Fig. 3: Multi-time success probability by MADDPG 2-partner

\section{B. Algorithms Comparison}

To show the advantage of MADDPG based cooperative sensing algorithm, we further compare the one-time success probability with random access and other DRL approaches in Fig. 4, including Q-learning and multi-agent DQN [3], [5], [7]. DQN takes use of multi-layer perceptron (MLP) instead of Q-table to map a state into an action. In the experiment, 
DQN MLP1 has one hidden layer with 300 neurons and DQN MLP2 has two hidden layers with with 300 neurons of each. Each algorithm is enforced two times with 1 partner and 2 partners respectively.

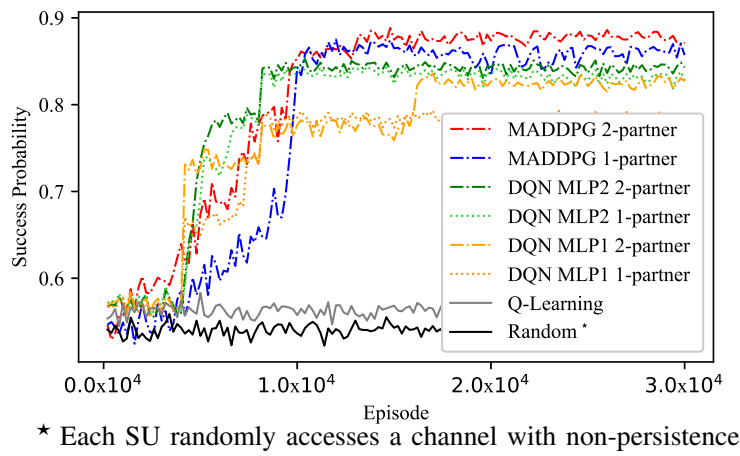

Fig. 4: One-time success probability comparison

As shown in Fig. 4, due to random access that stateless of the prior channel information, it shows the lowest success probability and can not been proved by episode. While channels belief fusion is effective for both MADDPG and DQN algorithm. With the help of 2 partners, the success probability enhances nearly 1\%, 2\% and 5\% for MADDPG, DQN MLP2 and DQN MLP1 with only 1 partner respectively. MADDPG with 2 partners shows the best sensing accuracy, which is in accordance with the results in Fig. 2.

However, Q-learning performs poorly in this scenario (actually it works well when there are only 2 SUs). Since the lack of other SU's observation, the state transition of each individual is time-varying and unreliable which makes the environment non-stationary. As the number of agents increasing, the fastgrowing size of Q-table makes the training difficult to ergodic all the possible environment status, and SUs are prevented to use the past experience reply in straight forward [8]. Thus Q-learning is not suitable to large-scale models.

\section{Impact of Transition Probability and False Alarm}

This section discusses the impact of Markov transition probability of channels and the false alarm probability which are uniformly distributed to the sensing accuracy.

Fig. 5(a) indicates that when $p_{0,0}$ changes from 0.3 to 0.9 , which means channels gradually incline to be occupied by PUs, and the vacant probability $p_{1,1}$ shrinks from 0.7 to 0.1 , the one-time success probability declines (for example, from $96 \%$ to approximately $70 \%$ in MADDPG) as the channels becoming busy. But compared with DQN MLP1 and MLP2, MADDPG still presents the advantage in sensing accuracy by at least $2 \%$ and $5 \%$ performance improvement respectively.

Suppose that SUs take the power detection threshold as the channels sense strategy, then there would exist a sensing error probability $p_{f a}$ to mistake a vacant channel $f$ as occupied (also known as false alarm), i.e. $p\left(\bar{b}_{n, f}=1 \mid O_{f}=0\right) \leq$ $p_{f a}, \forall n, f$. A smaller $p_{f a}$ declares that SUs have a larger probability to sense the channel correctly. But when $p_{f}$ is larger than 0.4, all DRL algorithms could not be well-trained due to too many sensing errors. Thus Fig. 5(b) only depicts the one-time success probability when $p_{f a}$ declines from 0.4 to 0 . The one-time success probability greatly increases along with
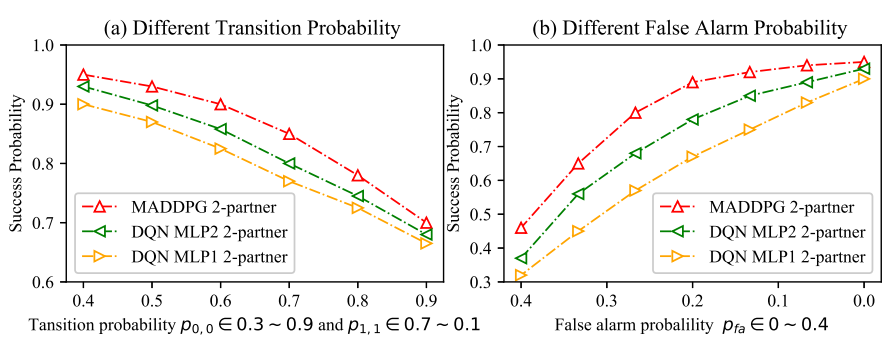

Fig. 5: One-time success probability with different Markov transition and false alarm probability

the reduction of sensing errors in both DQN and MADDPG algorithms. Similar to the previous results, compared with DQN MLP1 and MLP2, MADDPG still reveals its advantages on the sensing accuracy, especially when there exists a larger sensing error probability.

\section{CONCLUSION}

Although SUs can sense and take vacant channels for transmission opportunistically in CRNs, the network topology, channels state and PUs' action are still hard to predict. So a multi-agent reinforcement learning approach is proposed to enable each SU seek a vacant channel swiftly. In specific, the paper proposes a partners selection and channels state fusion algorithm to make each SU be global-aware of channels state and hence enhance the sensing accuracy. Furthermore, MADDPG is adopted to reduce the synchronization and communication overhead caused by the sensing cooperation of SUs for its centralized training and decentralized execution feature. Finally, the numerical simulation proves that the MADDPG based cooperative spectrum sensing scheme has great effectiveness and superiority in the one-time success probability compared with other main DRL algorithms.

\section{REFERENCES}

[1] R. Sarikhani and F. Keynia, "Cooperative spectrum sensing meets machine learning: Deep reinforcement learning approach," IEEE Commun. Lett., vol. 24, no. 7, pp. 1459-1462, 2020.

[2] T. Xu, T. Zhou, J. Tian, J. Sang, and H. Hu, "Intelligent spectrum sensing: When reinforcement learning meets automatic repeat sensing in 5g communications," IEEE Wirel. Commun., vol. 27, no. 1, pp. 46-53, 2020.

[3] J. Lundén, S. R. Kulkarni, V. Koivunen, and H. V. Poor, "Multiagent reinforcement learning based spectrum sensing policies for cognitive radio networks," IEEE J. Sel. Top. Sign. Proces., vol. 7, no. 5, pp. 858-868, 2013.

[4] S. K. Sharma and X. Wang, "Collaborative distributed q-learning for rach congestion minimization in cellular iot networks," IEEE Commun. Lett., vol. 23 , no. 4, pp. 600-603, 2019.

[5] S. Wang, H. Liu, P. H. Gomes, and B. Krishnamachari, "Deep reinforcement learning for dynamic multichannel access in wireless networks," IEEE Trans. Cogn. Commun.Netw, vol. 4, no. 2, pp. 257-265, 2018.

[6] T. P. Lillicrap, J. J. Hunt, A. Pritzel, N. Heess, T. Erez, Y. Tassa, D. Silver, and D. Wierstra, "Continuous control with deep reinforcement learning," in Proc. Int. Conf. Learn. Represent. (ICLR), Puerto Rio, May 2016.

[7] H. Chang, H. Song, Y. Yi, J. Zhang, H. He, and L. Liu, "Distributive dynamic spectrum access through deep reinforcement learning: A reservoir computing-based approach," IEEE Internet Things J., vol. 6, no. 2, pp. 1938-1948, 2019.

[8] R. Lowe, Y. Wu, A. Tamar, J. Harb, P. Abbeel, and I. Mordatch, "Multiagent actor-critic for mixed cooperative-competitive environments," in Neural Information Processing Systems (NeuralIPS), Long Beach CA, June 2017.

[9] W. Ning, X. Huang, K. Yang, F. Wu, and S. Leng, "Reinforcement learning enabled cooperative spectrum sensing in cognitive radio networks," J. Commun. Netw-s Kor, vol. 22, no. 1, pp. 12-22, 2020. 